\title{
A Color IMAGE BLIND Digital WATERMARKING ALGORITHM BASED ON QR CODE
}

\author{
Xuecheng Gong and Wanggen Li \\ School of Computer and Information, \\ Anhui Normal University, Anhui Wuhu, China
}

\begin{abstract}
With the rapid development of network technology and multimedia, the current color image digital watermarking algorithm has the problems of small capacity and poor robustness. In order to improve the capacity and anti-attack ability of digital watermarking. A color image blind digital watermarking algorithm based on QR code is proposed. The algorithm combines Discrete Wavelet Transform (DWT) and Discrete Cosine Transform (DCT). First, the color image was converted from RGB space to YCbCr space, and the Y component was extracted and the second-level discrete wavelet transform is performed; secondly, the LL2 subband was divided into blocks and carried out discrete cosine transform; finally, used the embedding method to embed the Arnold transform watermark information into the block. The experimental results show that the PSNR of the color image embedded with the QR code is 56.7159 without being attacked. After being attacked, its PSNR is more than 30dB and NC is more than 0.95. It is proved that the algorithm has good robustness and can achieve blind watermark extraction.
\end{abstract}

\section{KEYWORDS}

QR Code, Color Image, Arnold Transform, DWT

\section{INTRODUCTION}

With the development of the Internet, various digital products have appeared on the Internet. At the same time, with the rapid development of information digitization, there has also been a problem that copyright is not easy to protect [1]. Digital watermarking is a method to solve the problem of copyright protection. According to different embedding methods, it is divided into spatial domain watermark and transform domain watermark. The current digital watermark embedding into the vector transform domain better image can be improved watermark robustness and security [2].

The transform domain watermark technology embeds watermark information into the corresponding frequency coefficients through frequency domain transformation. Common methods include DWT and DCT. The low-frequency component embedding watermark through DCT transformation has strong robustness However, the anti-attack ability is weak[3]. The mixed use of DWT and DCT can balance the robustness and imperceptibility of the watermark image. Quick response code (quick response code) referred to as QR code, it can store a lot of information. Therefore, using QR code as a watermark can not only improve the robustness of the watermark, but also store more copyright information [4]. Arnold transform has periodicity and is widely used in image scrambling. Using its characteristics to transform QR code information can improve the security of watermarking [5].

David C. Wyld et al. (Eds): CoSIT, AIAPP, SIGL, CRIS, NLPML - 2021

pp. 67-75, 2021. CS \& IT - CSCP 2021

DOI: $10.5121 /$ csit.2021.110405 


\section{RELATED WORK}

He et al. [6] proposed a color image watermarking algorithm based on discrete wavelet transform, discrete cosine transform and singular value decomposition (DWT-DCT-SVD). First, convert the carrier image from RGB color space to YUV color space; then, perform a layer of discrete wavelet transform on the brightness component $\mathrm{Y}$, use discrete cosine transform to decompose the low frequency and divide it into blocks, and perform singular value decomposition on each block ; Finally, embed the watermark into the carrier image. However, there is a problem with the DWT-DCT-SCD method. A non-blind watermark image is needed to extract the watermark in the experiment and the watermark non-QR code is used in the experiment. Xu Jiangfeng et al. [7] proposed a digital watermarking scheme combining QR code, chaotic system and DWT-DCT. Carry out DWT operation on the carrier image and perform $4 \times 4$ block and DCT operation on the low frequency subbands. Then embed the QR code watermark through the chaotic system into the carrier image. The experimental results show that after the Gaussian noise attack, the PSNR value and NC value are low. The experiment uses gray-scale images, which is less practical. To solve the above problems, this paper proposes a color image blind digital watermarking scheme based on QR code. This scheme selects a color image as the carrier image, converts the RGB color space to the YCbCr color space, embeds the watermark into the luminance component Yand uses the Arnold transformation to encrypt the QR code. While improving the security and robustness of the watermark. It also increased the amount of watermark informatione.

\subsection{Discrete Wavelet Transform}

In digital image processing, it is necessary to discretize continuous wavelet and wavelet transform. Discretized wavelet and corresponding wavelet transform are called discrete wavelet transform [8]. Discrete wavelet transform is a spatio-temporal scale analysis method of information analysis theory and signal. It has multiple scales in the space and frequency domainand can continuously decompose images from low resolution to high resolution $[9,10]$. In addition, the DWT algorithm has a wide range of applications in the digital watermarking field. At present, many innovative and efficient joint algorithms related to DWT have been proposed.

In this paper, the RGB color space of the color carrier image is transferred to the YCbCr color space. Perform DWT transformation on Y component to obtain the horizontal and vertical low frequency LL, the horizontal low frequency and the vertical high frequency LH, the horizontal high frequency and the vertical low frequency HL, high-frequency components $\mathrm{HH}$ in the horizontal and vertical directions. DWT is performed on the LL subband again to obtain the low frequency component LL2. The low-frequency components represent image features. The highfrequency components represent the edges and details of the image. Embedding the watermark in the low frequency component LL2 can improve the robustness. As shown in Figure 1.

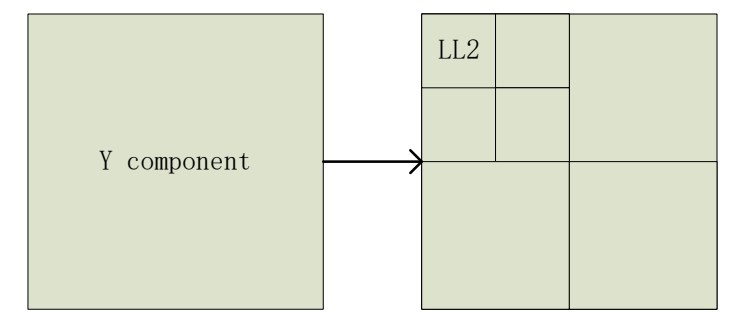

Figure 1. Wavelet decomposition diagram. 


\subsection{Discrete Cosine Transform}

Discrete Cosine Transform can transform the spatial domain signal into the frequency domain signal and has good decorrelation performance [11,12]. The important information of the image after discrete cosine transform is concentrated on the middle and low frequency coefficients. The position is the upper left corner of the DCT matrix, which has the ability to resist attacks. After being attacked, the embedded watermark information can still be extracted $[13,14]$. In this paper, the LL2 subband is divided into blocks, the blocks are subjected to DCT transformation, then the medium and low frequency coefficients are selected for watermark embedding. the DCT inverse transformation completes the image reconstruction.

\subsection{Arnold Transform}

The watermark image contains important information. The Arnold transform is used to scramble the image to achieve information encryption. At the same time, the Arnold transform is periodic. The number of scrambling can be used as the watermark key to further enhance its security [15]. The periodicity of Arnold transformation refers to the continuous transformation of the original image, the original image is obtained after $t$ times. The transformation period $t$ is related to the size of the image $\mathrm{M} \times \mathrm{N}$ [16]. The Arnold transformation is defined as follows:

$$
\left[\begin{array}{l}
x^{*} \\
y^{*}
\end{array}\right]=\left[\begin{array}{ll}
1 & 1 \\
1 & 2
\end{array}\right]\left[\begin{array}{l}
x \\
y
\end{array}\right](\bmod N)
$$

Among them, $(x, y)$ represents the pixel position before image scrambling, $\left(x^{*}, y^{*}\right)$ represents the image pixel location after scrambling, $\mathrm{N}$ represents the order of the image matrix.

QR code is a type of matrix two-dimensional code. It has many characteristics such as high-speed reading, high capacity, support for error correction processing, wide coding range, low costand easy production. Due to the high capacity of $\mathrm{QR}$ codes, using $\mathrm{QR}$ codes as digital watermarks can increase the information capacity. Because of its support for error correction processing, the robustness of the watermark can also be improved.

\section{EMBEDDing AND EXTRACTION OF WATERMARK}

This article uses QR code as a watermark which adds more information and improves the security of the watermark. The use of color images as carrier images is more widely used.

\subsection{Embedded Watermark}

(a) Convert the color carrier image from RGB color space to $\mathrm{YCbCr}$ color space according to the algorithm flow and extract the brightness component Y. (b) Perform a two-level DWT transformation on the luminance component to obtain the low frequency subband LL2, then implement $2 \times 2$ block division on the LL2 subband to obtain a block matrix. (c) Perform DCT transformation on each block ( $\mathrm{dct}=\mathrm{dct}(\mathrm{LL} 2)$ ) to obtain the transformed DCT matrix. Then extract the first value in the matrix from the DCT transformed block to form a new matrix F. (d) Use Arnold transform algorithm to scramble the original watermark image $\mathrm{W}$ to get the scrambled watermark $W^{*}$. (e) Embed the watermark $W^{*}$ into the matrix $\mathrm{F}$ using equations 2 and 3 to obtain the matrix $F^{*}$. Then replace each value of the matrix $F^{*}$ with the first value of each block in turn, and perform inverse DCT transformation on each block to obtain $L L 2^{*}$. 


$$
\begin{aligned}
& \lambda_{1}^{*}=\left\{\begin{array}{ll}
\lambda_{1}-T+3 \mathrm{a} / 4 & T \geq \mathrm{a} / 4 \\
\lambda_{1}-T-\mathrm{a} / 4 & \text { other }
\end{array} \quad W^{*}(\mathrm{i}, \mathrm{j})=0\right. \\
& \lambda_{1}^{*}=\begin{array}{ll}
\lambda_{1}-T+5 \mathrm{a} / 4 & T^{3} 3 \mathrm{a} / 4
\end{array} \boldsymbol{W}^{*}(\mathrm{i}, \mathrm{j})=1
\end{aligned}
$$

Among them, $T=\lambda_{1}$ moda, a is the embedding strength. Used to control the invisibility and robustness of embedded watermarks. $\lambda_{1}$ represents each value in each block matrix. $\lambda_{1}^{*}$ Represents the embedded value.

(f) Implement the second-level inverse DWT transformation on the obtained in the previous step to obtain the component. The brightness component of the embedded $\mathrm{QR}$ code is converted from the $\mathrm{YCbCr}$ color space to the RGB color space to obtain the color carrier image embedded in the QR code. Figure 2 is a flowchart of watermark embedding.

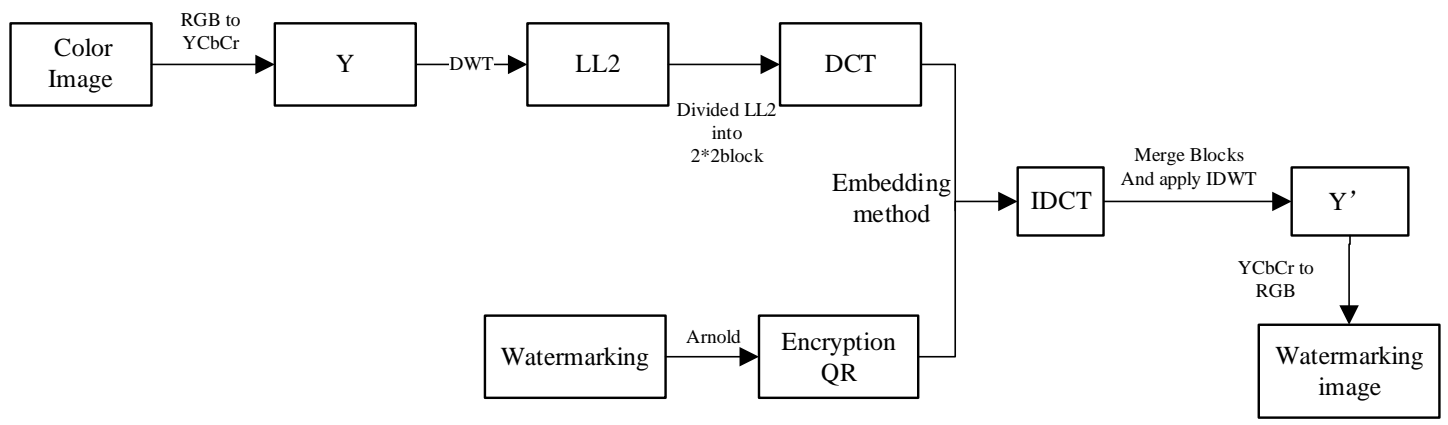

Figure 2. Watermarking embedding flow chart.

\subsection{Extract Watermark}

(a)First, convert the color carrier image embedded with QR code from RGB color space to $\mathrm{YCbCr}$ color space and extract the brightness component $Y^{*}$. (b) Perform two-level DWT transformation on the luminance component to obtain the low-frequency subband. Then perform $2 \times 2$ block on the subband. (c) Perform DCT transformation on each block to obtain the transformed DCT matrix. $\operatorname{dct}=\operatorname{dct}\left(L L 2^{*}\right)$. Then extract the first value in the matrix from the block after DCT transformation to form a matrix $F^{*}$. (d) The extraction of watermark information is the reverse process of watermark embedding. The watermark is extracted by equations 4 .

$$
W(\mathrm{i}, \mathrm{j})=\begin{array}{cc}
\text { 1 } & T>\mathrm{a} / 2 \\
\frac{1}{10} & \text { other }
\end{array}
$$

(e) According to the obtained $W^{*}$ in the previous step. Then perform Arnold transformation on it. Finally the watermark $\mathrm{W}$ is extracted. Figure 3 is a flow chart of watermark extraction.

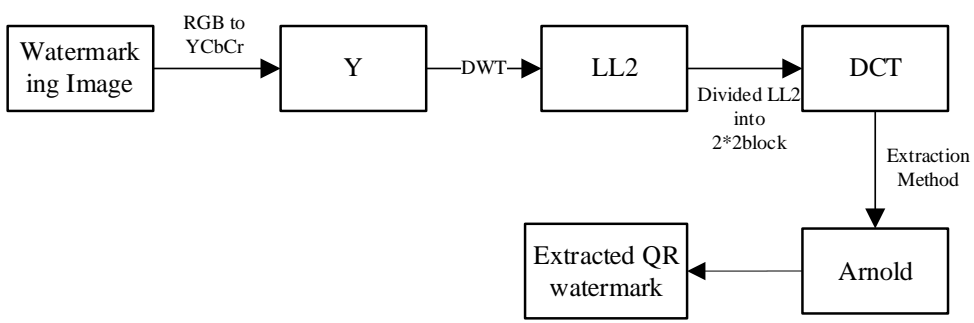

Figure 3. Watermarking extraction flow chart. 


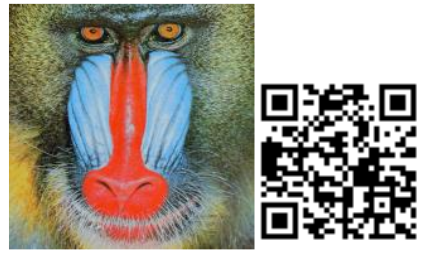

(a)Color Image (b)QR Code

Figure 4. Experiment Pictures.

\section{THE EXPERIMENTAL RESULTS}

The experimental environment is: Intel Core i5-4210M CPU; $2.60 \mathrm{GHz}$ frequency; Windows 10 64-bit operating system; Matlab2018a software. Select the color carrier image with $512 \times 512$ pixels, the $64 \times 64 \mathrm{QR}$ code is the watermark and the QR code carries the information. Figure 4(a) is a color carrier image, and Figure 4(b) is a QR code watermark image.

\subsection{Watermark Evaluation Standard}

Experiments usually use peak signal-to-noise ratio (PSNR) to measure the difference between the QR code-embeddsssed image and the unembedded original image. The greater the PSNR, the higher the recognition of the image embedded in the QR code with the original image. The definition of PSNR is:

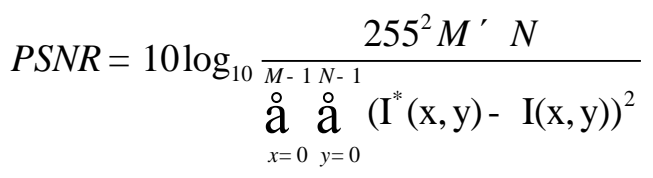

Among them, I $^{*}$ represents a watermarked color carrier image and I represents a color carrier image. When the PSNR value is greater, the color carrier image is closer to the watermarked color carrier image. The embedded watermark effect is better. PSNR $>30 \mathrm{~dB}$ usually means that the watermark is invisible and the image quality is better.

The normalized correlation coefficient (NC) represents the similarity between the original watermark and the extracted watermark. The $\mathrm{NC}$ value ranges from 0 to 1 . The closer the NC value is to 1 , the higher the similarity between the original watermark and the extracted watermark. The definition of $\mathrm{NC}$ is:

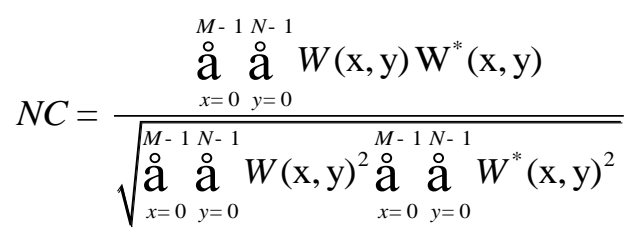

Among them, $\mathrm{W}$ represents the original watermark image. $\mathrm{W}^{*}$ represents the extracted watermark image.

\subsection{Analysis of Results}

In order to test the robustness of the algorithm, the experiment uses JPEG compression, noise filtering, cropping attack, rotation attack and median filtering to attack watermarked images. 
a) JPEG compression.

Table 1. JPEG Compression.

\begin{tabular}{|c|c|c|c|}
\hline who & 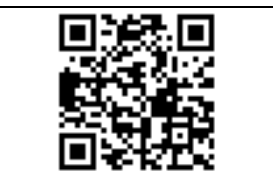 & & 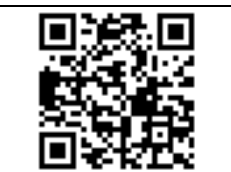 \\
\hline (a)JPEG(10\%) & (b)Extracted $(a=1)$ & (c)JPEG( $(80 \%)$ & (d)Extract $(\mathrm{a}=1)$ \\
\hline
\end{tabular}

In the experiment, the JPEG compression attack was performed on the image. Table 1(a)-(d) are the results of the JPEG compression attack under different factors. It can be measured that the extracted QR code can be recognized by the machine.

According to the data in Table 2, when the JPEG compression factor is $10 \%$, the PSNR value is 48.9158 and the $\mathrm{NC}$ value is 1 . At this time, the QR code can still be identified, indicating that the algorithm in this paper is robust against JPEG compression attacks and can ensure the integrity of the watermark information.

Table 2. JPEG Compression attack data.

\begin{tabular}{|c|c|c|c|c|c|c|}
\hline \multirow{2}{*}{ Methods } & \multicolumn{7}{|c|}{ Compression Ratio } \\
\cline { 2 - 7 } & $10 \%$ & $20 \%$ & $30 \%$ & $40 \%$ & $60 \%$ & $80 \%$ \\
\hline PSNR/dB & 48.9158 & 50.0294 & 50.6425 & 51.0356 & 51.6767 & 52.5922 \\
NC & 1 & 1 & 1 & 1 & 1 & 1 \\
\hline
\end{tabular}

b) Noise attack. The experiment uses salt and pepper noise and Gaussian noise attacks, as shown in Table 3.

Table 3. Noise attack.

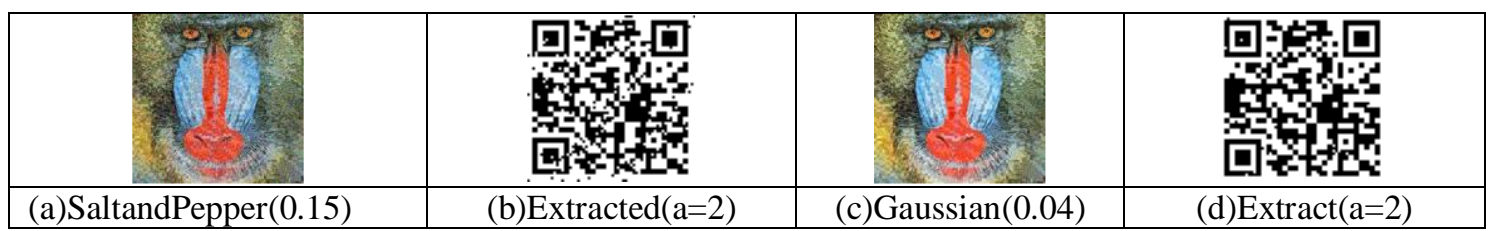

Table 4.Noise attack data.

\begin{tabular}{|c|c|c|c|c|c|c|c|}
\hline \multirow{2}{*}{ Method } & \multicolumn{3}{|c|}{ Salt and Pepper Noise } & \multicolumn{4}{c|}{ Gaussian Noise } \\
\cline { 2 - 8 } & 0.01 & 0.05 & 0.15 & 0.01 & 0.02 & 0.03 & 0.04 \\
\hline \multirow{2}{*}{ PSNR/dB } & 45.3121 & 41.7544 & 37.9263 & 43.0325 & 42.9243 & 42.7836 & 42.5694 \\
\hline NC & 1 & 1 & 09687 & 1 & 0.9997 & 09995 & 0.9962 \\
\hline
\end{tabular}

It can be seen from Table 4 that the PSNR value of the color carrier image embedded with the QR code is attacked by salt and pepper noise and Gaussian noise respectively. The PSNR value is also above $30 \mathrm{~dB}$. It indicating that the attacked carrier image shows strong robustness and can resist noise attack. From the perspective of the NC value, the extracted watermark maintains a high consistency with the original watermark and the extracted watermark can be identified.

c) Cropping attack. It can be seen from the different cropping areas in Table 6 that the QR code is embedded in the frequency domain of the carrier image.The cropping of different areas can still 
maintain a high NC value, the PSNR value can also be maintained above $30 \mathrm{~dB}$ and the QR code can be Identified. Therefore, this algorithm has good resistance to shearing attacks.

Table 5. Cropping attack.

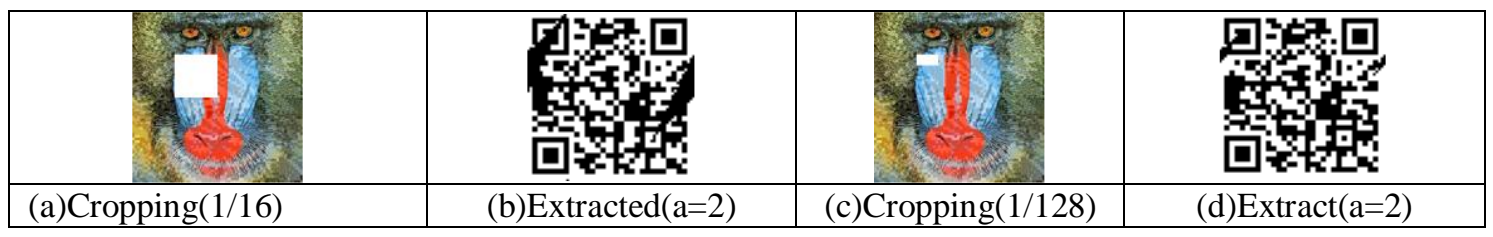

Table 6. Cropping attack data.

\begin{tabular}{|c|c|c|c|c|c|c|}
\hline \multirow{2}{*}{ Method } & \multicolumn{7}{|c|}{ Cropping Ratio } \\
\cline { 2 - 7 } & $1 / 8$ & $1 / 16$ & $1 / 32$ & $1 / 64$ & $1 / 128$ & $1 / 256$ \\
\hline PSNR/dB & 41.3916 & 41.3724 & 43.0706 & 45.3883 & 45.8662 & 46.0592 \\
\hline NC & 0.9600 & 0.9589 & 0.9782 & 0.9868 & 0.9928 & 0.9951 \\
\hline
\end{tabular}

d) Rotation attack and Median filtering. It can be concluded from Table 7 that the algorithm can resist rotation attacks and median filtering. The PSNR values are all above 30dB, and the NC values remain above 0.9. It shows that the algorithm has good robustness.

Table 7. Rotation attack and Median filtering data.

\begin{tabular}{|c|c|c|c|}
\hline \multirow{2}{*}{ Method } & \multicolumn{2}{|c|}{ Rotation Angle } & Median Filtering \\
\cline { 2 - 4 } & $5^{\circ}$ & $10^{\circ}$ & {$[3 \times 3]$} \\
\hline PSNR/dB & 42.4715 & 40.4322 & 45.2474 \\
\hline NC & 0.9693 & 0.9558 & 0.9991 \\
\hline
\end{tabular}

e) Comparison results. It can be seen from Table 8 that in the median filter attack, the algorithm in this paper is better than the non-blind QR code watermarking algorithm in the literature [7]. In this paper, the NC values of Gaussian noise and salt and pepper noise are both higher than 0.9. When the JPEG compression factor is $30 \%$, the NC value reaches 1, which is higher than the NC value in the literature [7]. It can be concluded that the algorithm in this paper is more robust than the algorithm in [7].

Table 8. Compare the results of different experiments.

\begin{tabular}{|c|c|c|}
\hline Attacks & $\begin{array}{c}\text { NC(Proposed } \\
\text { Method) }\end{array}$ & $\begin{array}{c}\text { NC(Reference } \\
\text { [7]) }\end{array}$ \\
\hline JPEG Compression30\% & 1 & 0.9875 \\
\hline Cropping 1/16 & 0.9589 & - \\
\hline Gaussian Noise 0.04 & 0.9962 & 0.9300 \\
\hline Salt and Pepper Noise 0.05 & 1 & 0.9849 \\
\hline Median Filtering [3×3] & 0.9991 & 0.9873 \\
\hline
\end{tabular}

\section{CONClusion}

In this paper, color images are used as carrier images and QR codes are used as watermark information to increase the watermark information carrying capacity, it also improves the watermark's anti-attack ability. Implementation of Arnold transformation on QR code, then implementation of DWT, DCT and block operations on carrier images. attack experimental data 
show that the algorithm in this article is resistant to JPEG compression, clipping attack, Gaussian noise, salt and pepper noise, median filtering, and rotation attacks. The PSNR values are all above $30 \mathrm{~dB}$. The algorithm proposed in this paper also has shortcomings. In a rotation attack, when the rotation angle exceeds $12^{\circ}$, the extracted $\mathrm{QR}$ code will not be recognized and information cannot be obtained through the device. In view of the above problems, the algorithm needs to be continuously improved.

\section{REFERENCES}

[1] P. Rasti \& S. Samiei, M.et al, (2016) "Robust non-blind color video watermarking using QR decomposition and entropy analysis,” J. Vis. Commun. Image Represent, vol. 38, no. 5, pp. 838-847.

[2] J. Wang \& Z. Du, (2019)“A method of processing color image watermarking based on the Haar wavelet,” J. Vis. Commun. Image Represent., vol. 64, pp. 102627.

[3] S. Kushlev \& R. P. Mironov, (2020) "Analysis for Watermark in Medical Image using Watermarking with Wavelet Transform and DCT,” 2020 55th Int. Sci. Conf. Information, Commun. Energy Syst. Technol, pp. 185-188, 2020.

[4] Bai TaoTao \& Liu Zhen et al, (2014) "Contourlet domain digital watermarking algorithm based on QR code,”Optoelectronics•Laser,vol. 25, no. 4, pp. 769-776.s

[5] Li Guohe \& Chen Chen,et al, (2018) "Digital watermark insertion and extraction method for QR code, ”Computer Engineering and Applications, vol. 55, no. 10, PP. 103-107, 114.

[6] Y. He \& Y. Hu, (2018) “A Proposed Digital Image Watermarking Based on DWT-DCT-SVD,” Proc. 2018 2nd IEEE Adv. Inf. Manag. Commun. Electron. Autom. Control Conf. 2018, pp. 1214-1218.

[7] Xu Jiangfeng \& Zhang Shouqiang, (2018) "DWT-DCT digital watermarking algorithm based on QR code,"Application Research of Computers, vol. 35, no. 5, pp. 1540-1544.

[8] Yuan Zihan \& Liu Decheng, et al, (2020) "New image blind watermarking method based on twodimensional discrete cosine transform," Optik (Stuttg), vol. 204, no. February 2020, pp. 164152.

[9] Zhu Jianzhong \& Yao Zhiqiang, (2014). "Color image blind watermarking algorithm based on DWTSVD and Turbo code,’Journal of Jilin University: Science Edition, vol. 52, no. 4 pp. 773-778.

[10] Y. A. Mekarsari \& D. R. I. M. Setiadi, et al, (2018) "Non-blind RGB image watermarking technique using 2-level discrete wavelet transform and singular value decomposition," 2018 Int. Conf. Inf. Commun. Technol, vol. 2018-Janua, pp. 623-627.

[11] M. Ali \& C. W. Ahn, et al, (2014) "A robust image watermarking technique using SVD and differential evolution in DCT domain," Optik (Stuttg), vol. 125, no. 1, pp. 428-434.

[12] Sunesh \& R.Rama Kishore, (2019) "A Novel and Efficient Blind Image Watermarking in Transform Domain,” Procedia Comput. Sci, vol. 167, no. 2019, pp. 1505-1514.

[13] D. O. Munoz-Ramirez \& V. Ponomaryov, et al, (2018) "A robust watermarking scheme to JPEG compression for embedding a color watermark into digital images," Proc. 2018 IEEE 9th Int. Conf. Dependable Syst. Serv. Technol, pp. 619-624.

[14] D. O. Muoz Ramirez \& V. Ponomaryov, et al, (2019) "Embedding a Color Watermark into DC coefficients of DCT from Digital Images,” IEEE Lat. Am. Trans, vol. 17, no. 8, pp. 1326-1334.

[15] Wu Fengbo \& Wang Feng, (2016) "Wavelet transform digital image watermarking algorithm based on HVS,"Applied Optics, vol. 35, no. 2, pp. 254-259.

[16] S. Saadi \& A. Merrad, (2019) "Novel secured scheme for blind audio/speech norm-space watermarking by Arnold algorithm,” Signal Processing, vol. 154, pp. 74-86. 


\section{AUTHORS}

Xuecheng Gong born in 1994, male, who studies at the Anhui Normal University, researching image processing.

Wanggen Li born in 1973, male, professor at the Anhui Normal University. His current research interests include biological computing and intelligent computing.
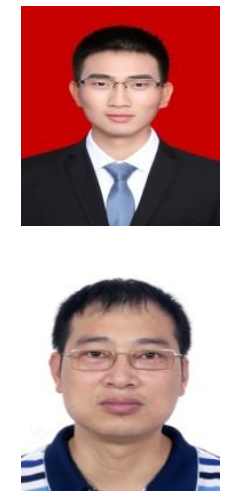

(C) 2021 By AIRCC Publishing Corporation. This article is published under the Creative Commons Attribution (CC BY) license. 